\title{
Could be the swine responsible of transmission to the humans of Dientamoeba fragilis infection?
}

\author{
Daniele Crotti', Marco Sensi ${ }^{2}$, Sonia Salamida², Silvia Crotti², Elisabetta Manuali ${ }^{2}$ \\ I L.P. in Parassitologia e Microbiologia Medica, Perugia \\ 2 Istituto Zooprofilattico dell'Umbria e delle Marche, Sede di Perugia
}

Key words: Diagnosis, Dientamoeba fragilis, Epidemiology, Giemsa stain, Swine

Il suino può essere responsabile della trasmissione all'uomo dell'infezione da Dientamoeba fragilis?

\section{SUMMARY}

Dientamoeba fragilis, atypical protozoon because "flagellate without flagella" but amoeba-like, of whom we know only the trophozoitic stage (an so its brittleness outside intestinal tract), is a frequent responsible of intestinal human infections, worldwide, and some authors relate that $D$. fragilis is the most frequent protozoon and parasite that can infects humans. Actually we don't know a sure potential "reservoir" in animals who are strictly in contact with humans, and it is difficult to understand its epidemiological chain, otherwise the transmissions to humans and from humans to humans. For all these reasons we performed another study on subjects of swine breedings, and among people who work in these breedings, that are in direct contact or not with pigs. Using standardized methodologies, we analyzed 224 faecal specimens of swine and 15 human specimens. We use for identification of $D$. fragilis the Giemsa stain. These were the results: $D$. fragilis was observed in $50.9 \%$ of pigs and $20 \%$ among humans $(30 \%$ in workers strictly in contact with breedings and pigs, $0 \%$ in familiars or other without a closed contact with swines). Other commensal protozoa were observed with variable associations, but in this article we want to analyze the possible transmission from this pigs to humans (and for us this protozoon is undoubtedly a "reservoir" of $D$. fragilis for humans), and underline two aspects: for the research of this protozoon, standard procedures area mandatory, with a permanent stain, as Giemsa stain, is necessary, and in all humans with various intestinal infections or troubles, particularly "irritable bowel syndrome" (or similar ones), the specimens must be analyzed for $D$. fragilis. At least we think that in the near future molecular studies are important for confirming this our observations, and for verifying eventual and probable differences inside genotypes of this very suggestive protozoon, that until now present not rarely phenotypic diversities.

\section{INTRODUZIONE}

Nel 2000 autori canadesi pubblicarono un articolo scientifico in cui si domandavano se i parassiti dei suini fossero un reale rischio di infezione per l'uomo (20). Tra i protozoi venivano riportati Giardia spp., Cryptosporidium spp., Toxoplasma gondii, Sarcocystis spp. e Blastocystis hominis, analizzandone una potenziale trasmissione zoonotica da questo animale all'uomo. Una ricerca condotta via Internet (http://www.k-state.edu/parasitology/classes/625protozoa26.html) ci permise di focalizzare quali fossero i protozoi che albergano o possono albergare il canale intestinale dei suini, sia come commensali che quali agenti potenzialmente responsabili di infezione sintomatica, e quindi vera e propria malattia. Venivano citati, in tale topic, soltanto Chilomastix mesnili, Enteromonas suis e Giardia duodenalis; stranamente non viene riportato Balantidium coli né altri a tutti noti potere essere presenti nel suino, in particolare i precedenti di cui sopra. Un interessante lavoro di tipo epidemiologico oltreché dia- gnostico, condotto e pubblicato da autori tedeschi nel 2006, metteva in evidenza la prevalenza delle infezioni protozoarie in giovani suini (lattonzoli, essenzialmente), riportando il rinvenimento, in un grosso numero di feci suine raccolte in 20 allevamenti diversi, dei seguenti protozoi: B. coli, Entamoeba spp., Iodamoeba spp., Isospora suis, Chilomastix spp., Eimeria spp. (12). Il medesimo lavoro riportava come non fossero mai stati reperiti Giardia spp., Trichomonas suis, Endolimax spp., Blastocystis spp., laddove se ne arguiva che $D$. fragilis non fosse stata di per sé mai adeguatamente ricercata. Un altro recentissimo lavoro del 2010 di un gruppo misto austriaco e ceco, che propone l'uso di determinati probes per il rilevamento in campo animale di protozoi dell'Ordine Trichomonadida rispetto agli altri, non riportava assolutamente il potenziale reperimento o meno di D. fragilis (18), evidentemente non prendendola assolutamente in considerazione, laddove $D$. fragilis è invece attualmente classificata, come Ordine, tra i Trichomonanida, e strettamente cor-

\section{Corresponding author: Daniele Crotti,}

Strada Comunale per Pilonico Paterno 4

06 I34 Perugia - Tel.: 075602372

E-mail: daniele.nene@email.it 
relata ad un altro appartenente a tale ordine, ossia Histomonas meleagridis, un flagellato responsabile di una grave patologia infettiva nel tacchino $(1,15)$.

Oggi noi sappiamo che $D$. fragilis è tutt'altro che infrequente nell'uomo, essendo stata correlata a diversificate sindromi intestinali, di natura o conatura infettiva, sia antecedentemente che successivamente al determinate lavoro di Johnson e collaboratori del 2004, nel quale descrivevano gli aspetti biologici, clinici e diagnostici di tale protozoo flagellato atipico (1), inquadrandolo e sottolineandolo quale emergente da una inspiegabile oscurità che datava da tempo (15). In tale review, il protozoo $D$. fragilis viene, da un punto di vista epidemiologico, riportato con frequenze a volte anche assai variabili in diversi Paesi europei ed extraeuropei, come in altri lavori specificamente pubblicati al riguardo, nel corso di questi ultimi dieci anni, sempre nell'uomo, e la cui presenza è fortemente correlata alla "sindrome del colon irritabile", ma non necessariamente soltanto a questa $(4,5,6,19,22,24,26,27)$.

In particolare traspare, in campo umano, come D. fragilis sia spesso presente, e non di rado D. fragilis sia o possa essere anche superiore, come prevalenze e/o incidenze, a Giardia duodenalis, da sempre ritenuta la causa più frequente di infezione sintomatica umana in ogni dove $(4,7,8,15,19,29)$.

Due sono peraltro gli aspetti che focalizzano tuttora gli studiosi riguardo la trasmissione, da uomo a uomo, di $D$. fragilis, dal momento che di questo "flagellato senza flagelli" si conosce solo la fase di trofozoita, ovvero non sembra esistere la fase cistica che ne garantirebbe la sopravvivenza in ambienti esterni anche sfavorevoli, e non si conoscevano sino a pochi anni addietro serbatoi animali degni di interesse, fatta salva la sua presenza nell'intestino di alcuni primati, nella fattispecie i gorilla, ove possono essere anche causa di malattia sempre a livello intestinale $(16,25)$. Alcune nostre recenti esperienze hanno peraltro messo in evidenza come non di rado $D$. fragilis possa ritrovarsi nelle feci di suini, soprattutto se in giovane età, ed in particolare negli allevamenti più o meno intensivi $(9,10,11)$. Per quanto concerne invece la trasmissione all'uomo, è stato più volte ipotizzata la sua trasmissione indirettamente attraverso un veicolo, che potrebbe essere rappresentato da alcuni elminti di coinvolgimento soprattutto umano, in particolare Enterobius vermicularis ed Ascaris lumbricoides (1, 2, 15, 17), sebbene altri lavori più recenti abbiano escluso tale possibilità (21).

Sulla base di tali premesse, riportiamo i risultati relativi ad un'indagine che si è svolta grosso modo nel corso del 2010, finalizzata, ancora una volta, alla ricerca di $D$. fragilis nel materiale fecale di suini d'allevamento, al fine di valutarne l'impatto potenziale epidemiologico, dal momento che laddove e quando il contatto tra suini e uomo è stretto, sembra aumentare la frequenza di reperimento di tale protozoo nelle feci umane, anche in assenza di manifestazioni apertamente o chiaramente patologiche $(7,9)$.

Verranno peraltro prese in considerazione anche le modalità strettamente diagnostiche (nei tempi e nei modi), dal momento che altre peculiarità di tale protozoo sono la sua "apparente" fragilità, una volta eliminato con le feci, e quindi la sua sopravvivenza in ambienti non adeguati, seppur non tutti siano d'accordo (13), e la necessità di ricorrere a specifiche colorazioni permanenti per confermarne la presenza con inequivocabile certezza dello stesso "flagellato ameboide" $(1,4,5$, $7,8,15,22,27)$.

Oggi giorno, negli ultimissimi tempi, sono state allestite tecniche di biologia molecolare, che potrebbero ovviare la difficoltà della diagnostica tradizionale, sempreché impossibilitati a farla e, al contempo, avendo a disposizioni risorse economiche adeguate $(3,22,23)$; questo quantomeno a nostro parere, fermo restando che è anche nostra intenzione pervenire ad un confronto diagnostico tra quello tradizionale che prevede almeno una colorazione specifica delle feci adeguata e quello in biologia molecolare, già in minima parte avviato (dati personali non pubblicati), utile anche per verificare analogie o differenze genetiche tra $\mathrm{i}$ ceppi isolati in campo umano ed in campo animale $(15,23,24,31)$, differenze genetiche già osservate in ceppi umani, applicando metodologie diversificate $(14,21,22,24,31)$.

\section{MATERIALI E METODI}

Per quanto riguarda i materiali, sono stati raccolti 224 campioni di feci suine in 11 diversi allevamenti della regione Umbria (1, in verità, già in provincia di Arezzo, e quindi regione Toscana, ma strettamente confinate con l'Umbria, al cui Istituto Zooprofilattico Sperimentale [IZS] fa usualmente riferimento). In tale presentazione non faremo riferimento, in quanto esula dal proposito voluto, alla diversa frequenza di reperimento di $D$. fragilis (e con essa di B. coli e/o altri protozoi, identificati o meno) all'interno degli allevamenti analizzati nonché ad altri fattori, in primis l'età o la categoria di appartenenza dei soggetti analizzati, per i quali rimandiamo a più idonee pubblicazioni in merito.

Il periodo dei campionamenti e dell'indagine immediatamente successiva è stato quello compreso tra giugno 2010 e gennaio 2011 . 
Una quota abbondante di materiale fecale veniva raccolta da un esperto veterinario vuoi nei box contenenti gli animali d'allevamento (le feci apparivano come appena o di recente emesse) vuoi direttamente dalla ampolla rettale del suino individuato a caso, come nelle precedenti indagini preliminari $(9,10,11)$.

La raccolta delle feci avveniva di mattino, solitamente tra le ore 9 e le ore 12, e quindi immediatamente portate al laboratorio dell'IZS, ove nel pomeriggio (o al massimo nelle prime ore del mattino successivo) venivano strisciate a gradiente su vetrino porta-oggetto, lasciate asciugare e quindi fissate con metanolo per un paio di minuti circa $(1,6,9)$. Conservati a temperatura ambiente, i vetrini venivano quanto prima colorati utilizzando la colorazione di Giemsa, da noi ritenuta più adatta per l'individuazione di $D$. fragilis $(1,4,5$, $7,8,10,11,13,15)$, sebbene altri autori, nei loro lavori pubblicati, così come nella loro routine diagnostica o di ricerca, utilizzino o possano utilizzare colorazioni estemporanee e/o permanenti diversificate $(3,12,15,22,23,25,29)$.

A colorazione avvenuta, entro alcuni giorni venivano eseguite da personale esperto le letture microscopiche, procedendo alla osservazione in immersione (obiettivo 100x) di almeno 200 campi microscopici, utilizzando un microscopio ottico tradizionale con applicata macchina fotografica idonea per le foto scattate sui preparati più significativi, registrando sia la positività sia valutandone una concentrazione media in termini qualitativi - semiquantitativi, in siffatta maniera: $5+=1 \mathrm{o}$ più trofozoiti di $D$. fragilis per almeno ogni $1-3$ campi microscopici (c. m.); $4+=1$ o più trofozoiti per almeno ogni $5-9 \mathrm{c}$. m.; $3+=1$ o più trofozoiti per almeno ogni $10-20 \mathrm{c} . \mathrm{m} . ; 2+=1$ o più trofozoiti per almeno ogni $30-50$ c. m.; $1+=1 \mathrm{o}$ più trofozoiti certi per almeno ogni $80-100$ c.m. I campioni venivano conservati anche in soluzione fisiologica, in formalina al $10 \%$, e in soluzione alcool $(50 \%)$ - PBS $(50 \%)$ in congelatore per eventuale future indagini biomolecolari.

I campioni conservati in formalina venivano successivamente osservati anche al microscopio ottico $(10 \mathrm{x}$ e $40 \mathrm{x})$ per valutare la presenza di $B$. coli ed eventuali elminti (uova e/o larve e/o altro), il cui interesse esula peraltro da codesto studio.

Non furono mai eseguite colorazioni all'acido resistenza per la ricerca eventuale di coccidi, Cryptosporidium spp. in particolare (1).

Anche per quanto riguarda tutti i protozoi, identificati o meno con le osservazioni condotte, è stata valutata un'ipotetica concentrazione, per la quale peraltro non riportiamo i dati in quanto lo riteniamo del tutto superfluo.

Al contempo sono stati raccolti, previa spiegazio- ne ed accettazione, 15 campioni di soggetti umani, corrispondenti a 4 diverse aziende, soggetti in parte operanti direttamente all'interno delle stalle e quindi a contatti frequenti diretti con $\mathrm{i}$ suini (10 soggetti) ed in parte mogli di allevatori o soggetti operanti negli uffici e quindi mai a contatto con i suini (5 soggetti).

I campioni di tali soggetti seguivano l'iter diagnostico analogo a quello percorso per i campioni dei suini.

\section{RISULTATI}

D. fragilis, alla colorazione di Giemsa, appare come una struttura ameboide, con bordi delicati, a volte contenente vacuoli o pseudovacuoli e/o inclusioni varie, spesso vagamente circolare ma talora irregolare, di dimensioni variabili (dai 4-7 micrometri ai 20 - 30, ed eccezionalmente oltre, micrometri). Presenta un aspetto delicato, a volta quasi diafano, insomma "fragile". Il Giemsa colora in azzurro (nelle sue varie tonalità: da azzurro intenso quasi blu ad un azzurro delicato sino a grigio-azzurro chiaro) il citoplasma, mentre i nuclei (più spesso due, non di rado 1 , ed eccezionalmente 4 , anche nel medesimo campione) vengono colorati in rosso (dal rosso vivo al rosso assai scuro quasi violetto); la peculiarità è che sempre o quasi sempre i nuclei sono frammentati in più parti e comunque mai compatti (vedi Figura I, Figura II, Figura III e Figura IV).

D. fragilis è stata reperita in 114 casi sui 221 analizzati, il che equivale al 50.9\%; le negatività per questo protozoo sono state del $45.1 \%$, mentre nel restante $4.0 \%$ la diagnosi non è stata fatta in quanto non si è potuto con certezza arrivare alla sicura identificazione di $D$. fragilis (vedi in parte Tabella 1). Questo a dire che la metà dei suini analizzati, o meglio, dei campioni fecali analizzati, è risultato positivo per la presenza, in bassa, media o alta concentrazione (vedi oltre) di $D$. fragilis.

Le frequenze di reperimento di altri protozoi, più spesso variamente associati, è riportata in Tabella 1: Endolimax nana è stata diagnosticata in 93 campioni $(41.5 \%$ del totale), B. hominis in 67 campioni (29.9\%), Iodamoeba buetschlii, tipica del maiale, un tempo denominata Iodamoeba suis, in $65(29.0 \%)$, il flagellato $C$. mesnili in 10 $(4.5 \%)$, E. suis, altro flagellato, in $10(4.5 \%)$, Entamoeba polecki (la cosiddetta "ameba del maiale") in $3(1.3 \%)$, mentre in 18 casi non si poté emettere un giudizio diagnostico definitivo. $B$. coli fu osservato in 97 campioni, pari al 43.3\%.

D. fragilis fu reperita nella stragrande maggioranza dei casi associata ad altro o ad altri protozoi; in 107 casi era così associata ad altri protozoi, pari al $93.9 \%$, mentre in soli 7 casi fu reperita da sola $(6.1 \%)$. 
Per quanto riguarda la presunta concentrazione di D. fragilis nei vari campioni fecali, come riportato sopra, è stata variabile con queste frequenze e questi numeri: $41.2 \%=1+(47$ casi $) ; 29.8 \%=2+$ (34 casi); $12.3 \%=3+(14$ casi) $12.3 \%=4+(14$ casi); $4.4 \%=5+(5$ casi $)$.

In tema di associazioni tra $D$. fragilis ed altri protozoi (osservate in 107 casi, come sopra detto), commensali o potenzialmente patogeni, di interesse suino e, anche, umano, la Tabella 2 è esplicativa al riguardo.

Per quanto concerne $B$. coli, da sempre noto come protozoo tipico dei suini, ed in tale indagine reperito in 97 casi (43.3\% dei campioni analizzati), è stato soprattutto reperito nella sua fase cistica (73 casi pari al $72.3 \%$ ), talora in entrambe le fasi sia cistica che trofozoitica (17 casi, pari al 17.5\%), mentre nella sola fase di trofozoite è stato osservato in 7 casi $(7.2 \%)$. In 81 casi $(27.2 \%)$ era associato a $D$. fragilis (eventualmente anche con altri protozoi), in 21 casi (9.4\%) era associato a uno o più protozoi diversi da $D$. fragilis, mentre in 15 casi $(6.7 \%)$ è stato reperito come unico protozoo. Per quanto riguarda i 15 campioni umani, 10 di soggetti a contatto con i suini e 5 non in contatto qual tale, le positività per $D$. fragilis sono state 3 , pari al 20.0\%; va però precisato che mentre nei 5 campioni di soggetti non a contatto diretto con i suini il risultato è sempre stato negativo (anche per altri protozoi eventuali), le 3 positività per $D$. fragilis sono state reperite soltanto nei soggetti a contatto più o meno diretto con i suini (pari quindi al $30.0 \%$ ). Va inoltre precisato che in 4 casi, sempre tra i soggetti a contatto con i suini, furono osservati 4 casi di $B$. hominis $(40.0 \%)$ e 2 casi di E. nana $(20.0 \%)$. In particolare in 1 caso $D$. fragilis era associata a $E$. nana ed in un altro a $B$. homi$n i s$, in 1 caso vi era un'associazione tra $E$. nana e $B$. hominis, in 2 casi era presente solo $B$. hominis. Essendo assai pochi i campioni fecali analizzati in campo umano, non riteniamo opportuno fare ulteriori precisazioni.

\section{DISCUSSIONE}

Da quanto riportato emerge come indubbiamente D. fragilis possa albergare l'apparato intestinale dei suini e attraverso le feci essere eliminata all'esterno, come già osservato in precedenti indagini, che tra l'altro riportano frequenze consimili $(9$, 10, 11). La frequenza complessiva, in tale lavoro, è stata, come detto e riportato in Tabella 1, del $50.9 \%$.

Anche altri protozoi sono stati reperiti nelle feci suine (Tabella 1), protozoi spesso riscontrabili, essenzialmente quali saprofiti, anche nelle feci umane, come $E$. nana $(41.5 \%$, e solitamente cisti a 2, 3 o 4 nuclei), B. hominis $(29.9 \%$, con la sua

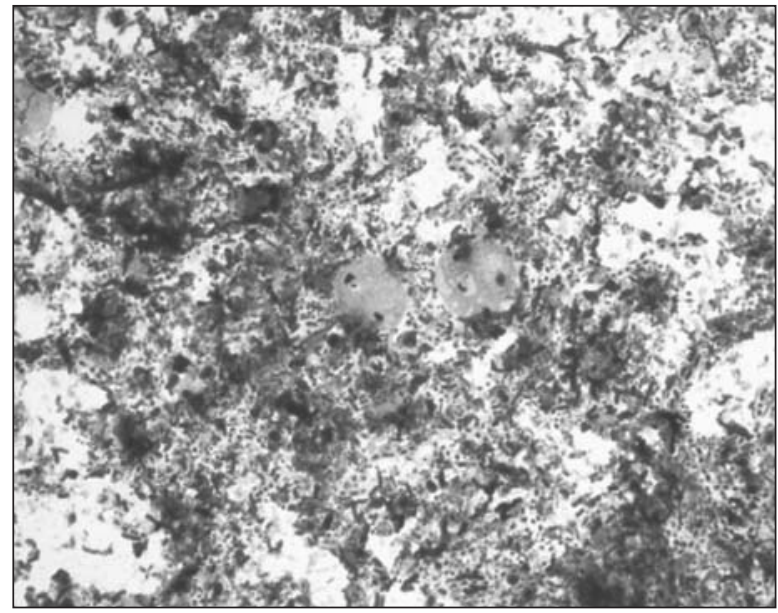

Figura I. D. fragilis suina con i tipici due nuclei citoplasmatici.

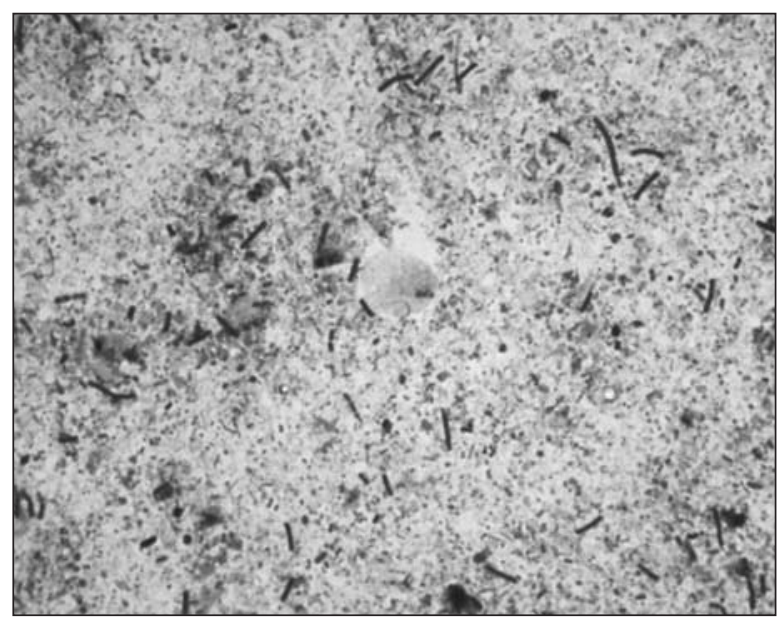

Figura II. Un trofozoite di D. fragilis suina, probabilmente ad un nucleo.

colorazione tipicamente rosacea di tutta la struttura vacuolare), I. buetschlii $(29.0 \%$, tipica del suino ma non frequente nell'uomo, con le sue forme cistiche piuttosto grandi e con il vacuolo iodoforo sempre ben evidente e ad 1 nucleo soltanto), e altri ancora.

$7 \mathrm{Ci}$ preme qui sottolineare come in un recente lavoro, di fatto una review, condotto a Singapore e pubblicato nel 2008, B. hominis o, meglio Blastocystis spp., venga rivalutata nella sua rilevanza clinica (28).

Ciò che peraltro ci stupisce in tale lavoro è che tale protozoo (ancora di incerta classificazione o forse rientrante nel "gruppo delle amebe") (1) venga confrontato con tanti altri, reperibili nelle feci umane, ma non venga presa in considerazione assolutamente $D$. fragilis, il che ci appare alquanto limitativo ed inidoneo.

D. fragilis, come detto nella parte introduttiva, è la causa più frequente di infezioni protozoarie e forse parassitarie in generale nell'uomo, rilevata sia da noi in precedenti indagini $(4,5,6,8)$, sia da 
svariati altri autori $(15,19,22,27)$; in ogni caso è spesso riscontrabile, seppur non sempre la più frequente, sempre per restare nel campo delle protozoosi patologiche $(15,29)$. La ricerca di $D$. fragilis è pertanto doverosa, pena la sottostima della stessa, nonché delle cause eziologiche delle diarree infettive $(24,26,27)$. Va inoltre considerato che usualmente la terapia per una giardiasi (e a maggior ragione per altre protozoosi responsabili di patologia vera e propria) è differente da quella applicata consuetamente per una giardiasi (30). Ecco quindi la necessità di ricercare, sempre e con dovizia, applicando le adeguate colorazioni permanenti (oltre quelle iniziali estemporanee), il protozoo ameboide $D$. fragilis $(1,8,15,29)$.

Il fatto di aver rilevato, in questa come in altre circostanze $(9,10,11)$, in campo suino, che circa il $50 \%$ dei suini, o comunque dei campioni fecali suini analizzati, sia e fosse stato positivo per $D$. fragilis, ci pone di fronte al fatto che $D$. fragilis possa trovare un serbatoio naturale in tale animale, e che lo stretto contatto, con modalità e/o per motivi vari, tra uomo e suino, sia causa di più elevate infezioni sostenute da $D$. fragilis in tale popolazione a rischio. Un'ipotesi che si potrebbe avanzare, ma andrebbe dimostrata, anche con metodologie diversificate, vuoi sin'anche biomolecolari, come accennato nell'introduzione, è quella che attraverso, o anche attraverso, l'areosolizzazione conseguente alla pulizia periodica degli ambienti chiusi ove i suini vengono allevati (quantomeno nei grossi allevamenti), i trofozoiti eliminati con le feci dagli animali possano essere ingeriti dall'operatore che compie siffatti lavori. Sta di fatto che laddove il contatto sia assai stretto tra uomo e suino, come in alcune realtà sudamericane, la frequenza di dientamoebiasi umana sia decisamente più alta che in altre situazioni $o$

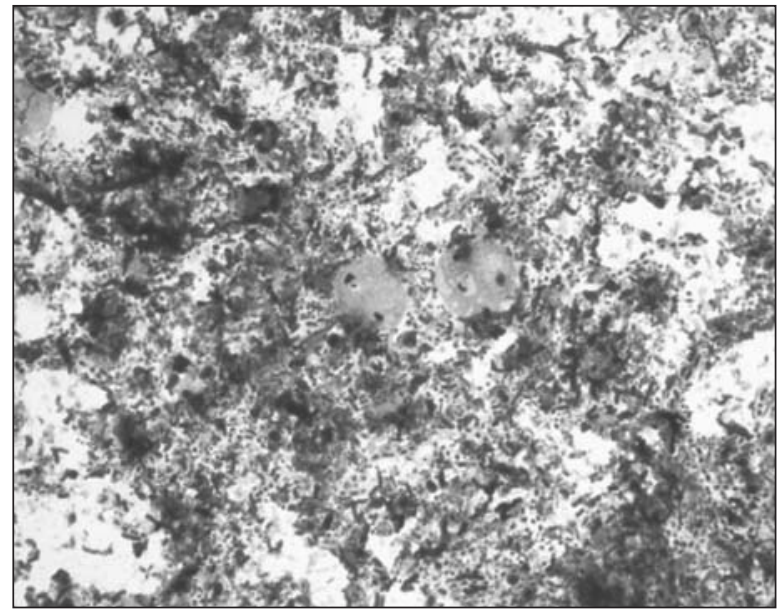

Figura III. D. fragilis suina a 2 e I nucleo.

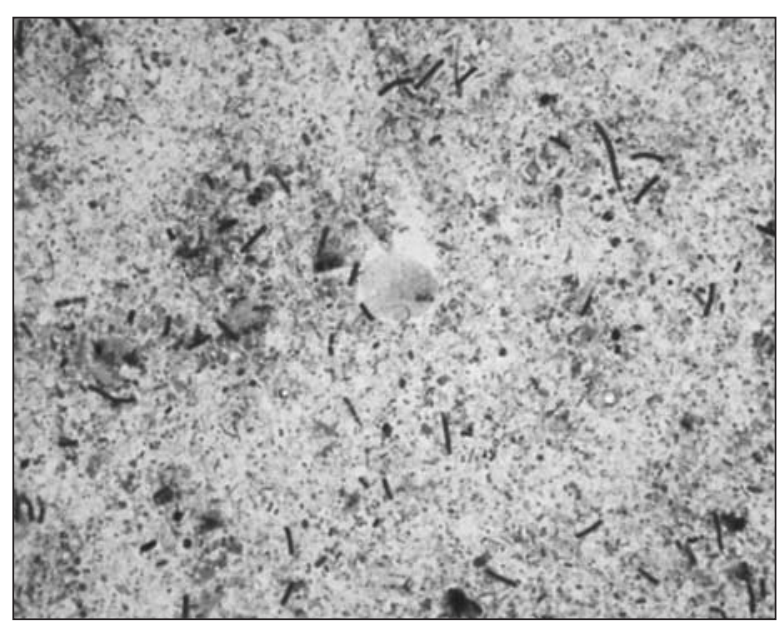

Figura IV. Sospetta se non verosimile D. fragilis suina a 4 nuclei.

realtà (7).

Resta tuttavia da domandarsi come mai invece, l'infezione da $B$. coli, sia di fatto ormai assente in

Tabella I. Prospetto generale risultati ottenuti rispetto a D. fragilis, in particolare, e ad altri protozoi. I campioni complessivi erano 224.

\begin{tabular}{lccccccccc} 
& D. fragilis & E. nana & B. hominis & I. buetschlii & C. mesnili & E. suis & E. polecki & $\mathrm{N}$. I. & B. coli \\
\hline $\mathrm{N}^{\circ}$ positivi & $\mathrm{II} 4$ & 99 & 67 & 65 & 10 & 10 & 3 & 18 & 97 \\
\hline \% positivi & 50.9 & 41.5 & 29.9 & 29.0 & 4.5 & 4.5 & 1.3 & $\mathrm{NV}$ & 43.3 \\
\hline
\end{tabular}

Note: N. I. = non identificato/non identificabile; Nv: non valutabile (in quanto I o più di I per campione fecale).

Tabella 2. Frequenze di reperimento di D. fragilis associata ad altri protozoi

\begin{tabular}{|c|c|c|}
\hline D. fragilis + B. hominis + E. nana + I. buetschlii & 3 casi & $2.8 \%$ \\
\hline D. fragilis $+B$. hominis + E. nana & 21 casi & $19.6 \%$ \\
\hline D. fragilis + B. hominis + I.buetschlii & 5 casi & $4.7 \%$ \\
\hline D. fragilis $+I$. buetschlii + E. nana & 21 casi & $19.6 \%$ \\
\hline D. fragilis $+B$. hominis & 18 casi & $16.8 \%$ \\
\hline D. fragilis + E. nana & 30 casi & $28.0 \%$ \\
\hline D. fragilis + I. buetschlii & 9 casi & $8.5 \%$ \\
\hline
\end{tabular}

NB: non viene riportata la presenza o meno di B. coli, vuoi in associazione con D. fragilis e/o altri protozoi (vedi testo); in tutti i casi non vengono segnalate le rare osservazioni di protozoi non identificabili o non identificati. 
campo umano, come da più lavori e da più parti riferito $(3,4,26,27,29)$, laddove ancora frequente sembra essere in campo suino, come il presente lavoro sottolinea, anche se altrove e forse con catene epidemiologiche diversificate può ancora talora reperirsi anche in campo strettamente medico (7).

Per concludere, riteniamo assai probanti queste nostre ulteriori osservazioni che, se da un lato indicherebbero nel suino una fonte importante di contagio per l'uomo relativamente a $D$. fragilis (come per altri protozoi non patogeni), dall'altro si impone sempre, in situazioni cliniche relative all'apparato gastroenterico, la ricerca di tale protozoo con tecniche tradizionali, innanzitutto, e/o con tecniche più moderne, in tutti i campioni fecali onde evitare una sua sottostima, anche pericolosa; a dire pure che una conoscenza anamnestica non soltanto tradizionale, ma, se ci permettete il termine, anche storica e geografica, potrebbe essere assai utile per meglio intraprendere un percorso diagnostico parassitologico razionale, efficiente ed efficace, quando necessario $(1,3,8,10$, $13,19,22,23,24)$.

Interessante e suggestiva infine è la possibilità, futura, anche se di un futuro già iniziato, di applicare metodologie moderne, di natura essenzialmente biomolecolare, che può permettere la conferma, l'identificazione, la differenziazione di diversi genotipi o comunque "varianti genotipiche" (nonché fenotipiche, ma per questo non crediamo siano necessarie particolari attrezzature se non quelle convenzionali, purché con "scienza, coscienza e conoscenza" utilizzate ed applicate) in tale protozoo ormai alla ribalta clinica da alcuni anni: D. fragilis (3, $14,15,21,23,26,30,31)$.

\section{BIBLIOGRAFIA}

1. Bernieri F, Crotti D. Infezioni da protozoi. Microbiologia Medica 2001; 16 (I): 98-108

2. Burrows RB, Swerdlow MA. Enterobius vermicularis as a Probable Vector of Dientamoeba fragilis. 1956; 5 (2): 258-65.

3. Calderaro A, Gorrini C, Montecchini S, et al. Evaluation of a real-time polymerase chain reaction assay for the detection of Dientamoeba fragilis. Diag Microb Infect Dis 2010; doi: 10.1016/j.diagmicrobio.2010.02.016.

4. Crotti D, D'Annibale ML. Dientamoeba fragilis: aspetti di parassitologia clinica e diagnostica di laboratorio. Parassitologia 2001; 43: 135-8.

5. Crotti D, D'Annibale ML. Dientamoena fragilis: suo ruolo nelle infezioni intestinali umane. Infez Med 2007; 1: 30-9.

6. Crotti D, D’Annibale ML. Attualità in tema di infezioni intestinali causate da Dientamoeba fragilis e Giardia duodenalis. Rec Progr Med 2007; 98 (6): 1-6.

7. Crotti D, D’Annibale ML, Basileo M, La Torre G. Sondaggio preliminare copro-parassitologico in una zona andina del Perù. Infez Med 2007; 3: 181-6.

8. Dientamoeba fragilis is more prevalent than Giardia duodenalis in children and adults attending a day care centre in Central Italy. Parasite 2005; 12: 165-70.

9. Crotti D, Manuali E, Sensi M, Salamida S, Vneditti G, Crotti S. Potenziale ruolo del Suino quale serbatoio di Dientamoeba fragilis, protozoo responsabile della dientamoebiasi umana. Il Progresso Veterinario 2007; 11: 501-3.

10. Crotti D, Sensi M, Crotti S, Grelloni V, Manuali E. Dientamoeba fragilis in swine population: A preliminary investigation. Vet Parasitol 2007; 145: 349-51.

11. Crotti D, Sensi M, Manuali E, Crotti S. Dientamoeba fragilis nella popolazione suina: indagini preliminari. Microb Med 2007; 4: 321-3.

12. Damriyasa M, Bauer C. Prevalence and age-dependent occurrence of intestinal protozoon infections in suckling Piglets. Berl Muench Tierärztl Wochenschr 2006; 119 (7/8): 287-90.

13. De Canale E, Tessari A, Campion L, Rossi L. Dientamoeba fragilis: è realmente fragile? Approccio al Trattamento del campione e diagnosi rapida al microscopio. Parassitologia 2003; 45: 19-22.

14. Johnson JA, Clark CG. Cryptic Genetic Diversity in Dientamoeba fragilis. J Clin Microbiol 2000; 38 (1): 4653-4.

15. Johnson EH, Windsor JJ, Clark CG. Emerging from Obscurity: Biological, Clinical, and Diagnostic Aspects of Dientamoeba fragilis. CMR 2004; 17 (3): 553-70.

16. Lank ester F, Kiyang JA, Bailey W, Unwin S. Dientamoeba fragilis: initial evidence of parhogenicity in the western lolawnd gorilla (Gorilla Gorilla Gorilla). J Zoo Wild Med 2010; 41(2): 350-2.

17. Menghi CI, Makiya R, Gatta CL, Méndez OC. Dientamoeba fragilis: técnicas moleculares para dilucidar su modo de transmisión. Parasitol Latinoam 2005; 60: 25-31.

18. Mostegl MM, Richter B, Nedorost N, et al. Design and validation of an oligonucleotide probe for the detection of Protozoa from the order Trichomonadida using chromogenic in situ hybridization. Vet Parasit 2010; 171: 1-6.

19. Norberg A, Nord CE, Evengård B. Dientamoeba fragilis - a protozoal infection which may cause severe bowel Distress. CMI 2003; 9: 65-8.

20. Olson ME, Guselle N. Are Pig Parasites a Human Health Risk? Adv Pork Prod 2000; 11: 153-61.

21. Stark D, Barrat J, Ellis J, Harkness J, Marriott D. Repeated Dientamoeba fragilis infections: a case of two families from Sydney, Australia. Inf Dis Reports 2008; 1: e4

22. Stark D. Barratt J, Roberts T, Marriott D, Harkness J, Ellis J. A Review of the Clinical Presentation of Dientamoebiasis. Am J Trop Med Hyg 2010; 82 (4): 614-9.

23. Stark D, Beebe N, Marriott D, Ellis J, Harkness J. Detection of Dientamoeba fragilis in fresh stool specimens Using PCR. Int J Parasitol 2005; 35: 57-62.

24. Stark D, Beebe N, Marriott D, Ellis J, Harkness J. Dientamoebiasis: clinical importance and recent advances. Trends In parasitology 2006; 22 (2): 92-6

25. Stark D, Phillips O, Peckett D, et al. Gorillas are a host for Dientamoeba fragilis: An update on the life cycle and host distribution. Vet Parasitol 2008; 151: 21-6.

26. Stark D, van Hal S, Marriott D, Ellis J, Harkness J. Irritable bowel syndrome: A review on the role of intestinal Protozoa and the importance of their detection and diagnosis. Int J Parasitol 2007; 37: 11-20.

27. Stensvold CR, Arendrup MC, Mølbak K, Nilsen HV. The prevalence of Dientamoeba fragilis in patients 
with Suspected enteroparasitic disease in a metropolitan area in Denmark. CMI 2007; 13: 839-42.

28. Tan KSW. New Insights on Classification, Identication, and Clinical Relevance of Blastocystis spp. CMR 2008; 21(4): 639-65.

29. Vandenberg O, Peek R, Souayah H, et al. Clinical and microbiological features of dientamoebiasis in patients Susoected of suffering from a parasitic gastrointestinal illness: A comparison of Dientamoeba fragilis and Giardia lamblia infections. Int $J$ Infec Dis 2006; 10: 255-61.

30. Vandenberg O, Souaya h H, Mouchet F, Dediste A, van Gool T. Treatment of Dientamoeba fragilis infection with Paromomycin. Ped Infect Dis J 2007; 26 (1): 88-90.

31. Windsor JJ, Clark CG, MacFarlane L. Molecular typing of Dientamoeba fragilis. Brit $J$ Biomed Sciences 2004; 61(3): 153-55. 